\title{
Acknowledgement to Reviewers of International Journal of Neonatal Screening in 2018
}

International Journal of Neonatal Screening Editorial Office

MDPI, St. Alban-Anlage 66, 4052 Basel, Switzerland

Published: 9 January 2019

\begin{abstract}
Rigorous peer-review is the corner-stone of high-quality academic publishing. The editorial team greatly appreciates the reviewers who contributed their knowledge and expertise to the journal's editorial process over the past 12 months. In 2018, a total of 37 papers were published in the journal, with a median time to first decision of 20 days and a median time to publication of 49 days. The editors would like to express their sincere gratitude to the following reviewers for their cooperation and dedication in 2018:
\end{abstract}

\author{
Aithal, Sreedevi \\ Al-Dirbashi, Osama \\ Andersson, Hans \\ Angastiniotis, Michael \\ Bailey, Donald B. \\ Bain, Barbara J. \\ Bierle, Craig \\ Bonham, Jim \\ Bubbico, Luciano \\ Butcher, Emma \\ Caprari, Patrizia \\ Chien, Yin-Hsiu \\ Cornel, Martina \\ Cortés Castell, Ernesto \\ Coutinho, Maria Francisca \\ Daniel, Yvonne A. \\ Dejaco, Daniel \\ Dotsikas, Yannis \\ Eckman, James R. \\ Engel, Paul C. \\ Etchegary, Holly \\ Ewer, Andrew \\ Feucthbaum, Lisa \\ Fuente, Adrian \\ Gardner-Berry, Kirsty \\ Gelb, Michael \\ Giraldo, Pilar \\ Grosse, Scott D. \\ Haas, Nikolaus \\ Hammarström, Lennart \\ Henderson, Matthew \\ Hinton, Cynthia F. \\ Hokanson, John Smith
}

Hom, Lisa A.

Hoppel, Charles L.

$\mathrm{Hsu}$, Lewis

Inusa, Baba

Jansen, Marleen

Jerebtsova, Marina

Kay, Denise M.

Kennedy, Colin

Kunz, Joachim

Lambert, Chris

Leo, Carlo Giacomo

Loeber, J. Gerard

Lorey, Fred

Mak, Chloe Miu

Matern, Dietrich

McGill, James J.

McGrath, Andrew P.

McPherson, Bradley

Mercer, Kristina

Moser, Ann B.

Nagao, Masayoshi

Newton, Valerie

Nicholls, Stuart G.

Olivieri, Antonella

Orsini, Joe

Pasquali, Marzia

Pastores, Gregory M.

Pekrun, Arnulf

Poulakis, Zeffie

Powell, Cynthia M.

Price, Patricia

Pulignani, Silvia

Ralli, Massimo 
Rausch, Christopher M.

Riede, Frank-Thomas

Rinaldo, Piero

Saadallah, Amal

Sequí-Canet, José Miguel

Sirdah, Mahmoud

Sommerburg, Olaf

Sontag, Marci

Stamminger, Thomas

Steyger, Peter

Stove, Christophe P.

Streetly, Allison

Tavakoli, Norma P.
Therrell, Bradford

Tortorelli, Silvia

Uilenburg, Noëlle

Van Trotsenburg, Paul

Van Zanten, Gijsbert A.

Veraguth, Dorothe

Von Döbeln, Ulrika

Vona, Barbara

Webster, Dianne

Wilcken, Bridget

Wiley, Veronica

Wilson, Callum

Yoshinaga-Itano, Christine

(C) 2019 by the author. Licensee MDPI, Basel, Switzerland. This article is an open access article distributed under the terms and conditions of the Creative Commons Attribution (CC BY) license (http://creativecommons.org/licenses/by/4.0/). 